\title{
The Orbital Elements of RZ Comae
}

\author{
L. BINNENDIJK \\ Flower and Cook Observatory, University of Pennsylvania, Philadelphia, Pennsylvania
}

(Received 4 November 1963)

\begin{abstract}
Photoelectric observations made by Broglia and spectroscopic observations made by Struve and Gratton have been used to determine new absolute orbital elements for RZ Comae. A change in the shape of the light curve can be explained by a subluminous region located on the larger star.
\end{abstract}

\section{INTRODUCTION}

A CCURATE photoelectric observations of RZ Comae were made in yellow light by Broglia (1960) during the year 1958 at the Merate Observatory in Italy. Two light curves were observed within a period of four months. The first light curve was obtained within the interval JD 2436220-285, and the second light curve within the interval JD 2436310-341. The light elements are:

JD Hel. Min. $\mathrm{I}=2434837.4198+0.3385060 \mathrm{E}$.

The shapes of the light curves differ. Primary minimum remains the same. However, the first light curve is about 0.02 fainter during secondary minimum and during both maxima than the second light curve. An interval of constant light during primary minimum indicates a total eclipse. The depths of the eclipses are well determined and reliable orbital elements should be expected from these complete eclipses.

There are indications that the published orbital elements are not correct, however. Broglia obtained an individual value of $k$ from the shape relation of each of the four eclipse curves. These four values of $k$ are listed in Table VI of his publication. Their mean value is $k=0.80$. Additional data given in this table were used to check this value. The phase angles at external and internal tangency indicate the durations of the four eclipses to be different. Since a circular orbit was assumed, mean values of these phase angles were determined. For Broglia's assumed inclination of $90^{\circ}$ a value of $k=0.75$ was found. The depth relation of each rectified light curve affords another check on the value of $k$. By using the data in Table V of Broglia's publication and his assumed limb darkening, a mean value of $k=0.61$ was obtained. The disagreement among these three mean values of $k$ is too large.

\section{LIGHT IN THE MAXIMA}

It was decided to repeat the orbital determination. First the normal points in Broglia's Tables IIIa and IIIb were normalized. New phases, counted from primary minimum, were obtained by subtracting

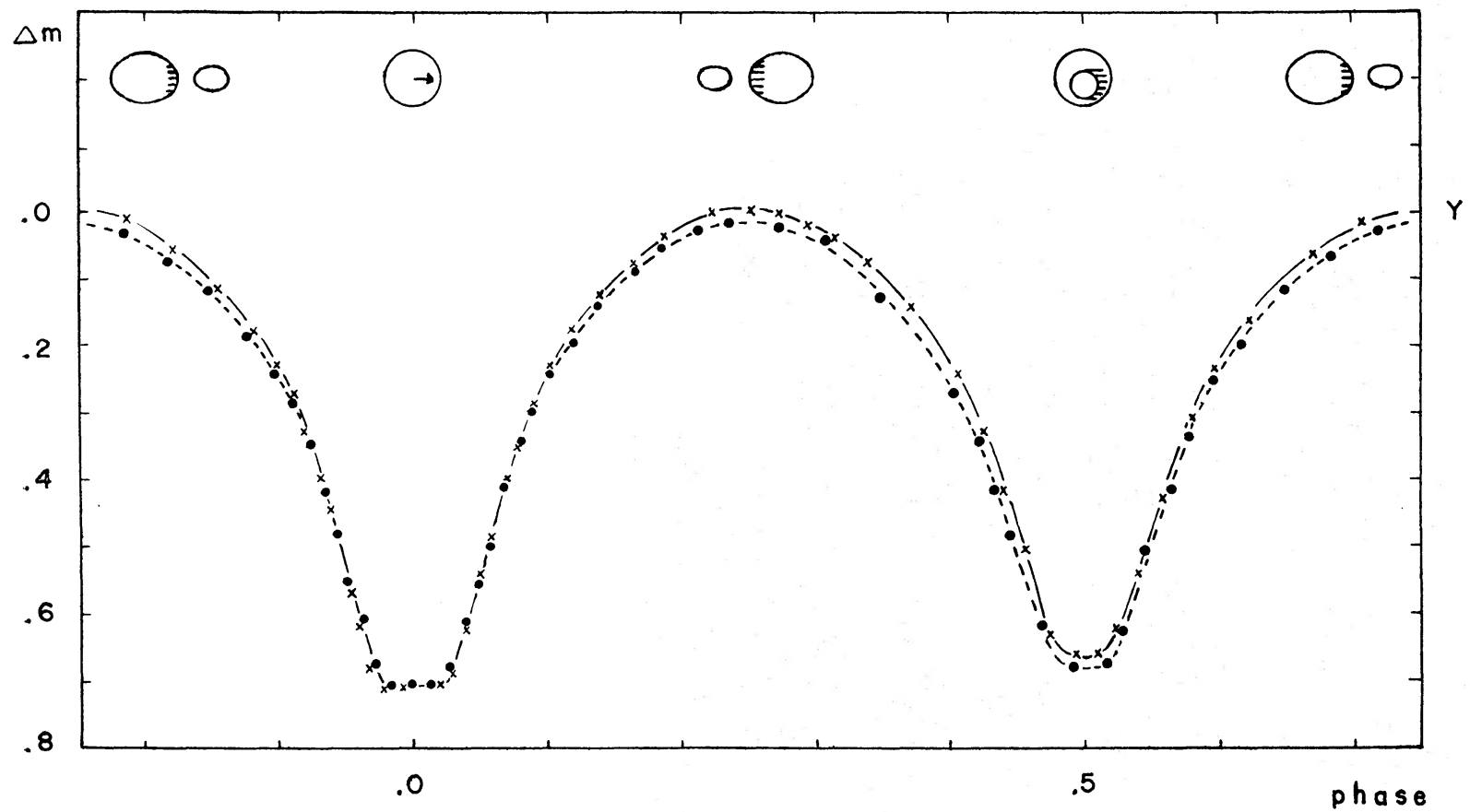

Fig. 1. Normal points of RZ Comae according to Broglia. The dots refer to the first light curve (JD 2436220-285), the crosses refer to the second light curve (JD 2436310-341). The curves are the theoretical light curves derived from new orbital elements. A subluminous region on the larger star can explain the differences between the light curves. 
0 ? 4977 from the phases listed by Broglia. A correction of $+0{ }^{\mathrm{m}} \cdot 140$ was applied to the magnitude differences listed by him so that the maximum of the second light curve became equal to zero. The normalized magnitudes and phases are plotted in Fig. 1. The dots refer to the first light curve and the crosses to the second one. These magnitudes and phases were converted into intensities and phase angles. Least-squares solutions were made of the light in the maxima according to the Fourier formula :

$$
l=A_{0}+A_{1} \cos \theta+A_{2} \cos 2 \theta+B_{1} \sin \theta+\mathrm{B}_{2} \sin 2 \theta .
$$

The coefficients and probable errors are listed in Table I. The coefficient $A_{1}$ has the sign predicted by theory. The coefficients $B_{1}$ and $B_{2}$ are small.

\section{ORBITAL ELEMENTS}

Orbital elements were determined from the second light curve (JD 2436310-341) for two reasons. First the small probable errors of the Fourier coefficients indicate the light in the maxima to be well defined. In addition, the depths of the minima of this light curve are unequal. This is favorable for determining the reflection coefficients.

The rectification process was carried out in the usual way. First the sine terms were removed by subtraction. Next the reflection coefficients were determined in order to rectify the intensities for reflection. They were found in the following way. The Fourier formula was extrapolated to $\theta=0^{\circ}$ and $\theta=180^{\circ}$. Provisional depths were found by differencing each of these values with the intensity at corresponding minimum. The ratio of the reflection coefficients was obtained from the ratio of these depths. The difference of the reflection coefficients was found from $A_{1}$. The reflection coefficients were then determined separately and their mean value was forthcoming. The rectification for oblateness concluded the process for the intensities. Finally the phase angles were rectified.

It was found that a coefficient of limb darkening $x=0.4$ was most satisfactory. The depth relation required $k=0.54$ for a grazing eclipse and $k=0.525$ for a central eclipse. The shape relation yielded $k=0.53$ for primary minimum and $k=0.54$ for secondary minimum. The following set of orbital elements was obtained from a consistent set of parameters. Primary minimum is the total eclipse.

$$
\begin{array}{lll}
x=0.4 & k=0.53 & \alpha_{0}{ }^{\mathrm{tr}}=1.027 \\
a_{g}=0.48 & a_{s}=0.26 & \epsilon=0.15 \\
b_{g}=0.41 & b_{s}=0.22 & i=81.1 \\
L_{g}=0.750 & L_{s}=0.250 & I_{g}=0.843 I_{s}
\end{array}
$$

In this list $\epsilon=(a-b) / a$ is the oblateness, $i$ is the

\begin{tabular}{|c|c|c|c|c|c|}
\hline No. & $A_{0}$ & $A_{1}$ & $A_{2}$ & $B_{1}$ & $B_{2}$ \\
\hline 1. & $\begin{array}{l}+0.8566 \\
\pm \quad 42\end{array}$ & $\begin{array}{l}-0.0044 \\
\pm \quad 35\end{array}$ & $\begin{array}{l}-0.1266 \\
\pm \quad 54\end{array}$ & $\begin{array}{l}+0.0031 \\
\pm \quad 14\end{array}$ & $\begin{array}{l}-0.0013 \\
\pm \quad 20\end{array}$ \\
\hline 2. & $\begin{array}{r}+0.8627 \\
\pm \quad 18\end{array}$ & $\begin{array}{r}-0.0039 \\
\pm \quad 13\end{array}$ & $\begin{array}{l}-0.1402 \\
\pm \quad 24\end{array}$ & $\begin{array}{l}-0.0009 \\
\pm \quad 6\end{array}$ & $\begin{array}{l}-0.0020 \\
\pm \quad 8\end{array}$ \\
\hline
\end{tabular}
inclination corrected for polar flattening, and $I$ is the mean surface brightness of the individual components. Table II lists the phases and observed magnitudes of
TABLE I. Light in the maxima.

the normals along with the number of observations contained in each normal. The observed values were compared with the theoretical values computed from the orbital elements and the resulting $O-C$ 's are

\begin{tabular}{|c|c|c|c|c|}
\hline Phase & $\begin{array}{c}\text { Obs. } \\
\Delta m\end{array}$ & $n$ & $O-C$ & $\underset{\Delta m}{\operatorname{Rect} .}$ \\
\hline 0.0166 & 0.714 & 7 & +0.001 & +0.313 \\
\hline 0.0270 & 0.692 & 6 & +0.001 & +0.301 \\
\hline 0.0379 & 0.628 & 6 & +0.001 & +0.256 \\
\hline 0.0479 & 0.552 & 5 & -0.005 & $\begin{array}{r}0.202 \\
\end{array}$ \\
\hline 0.0572 & 0.492 & 5 & 0.000 & $\begin{array}{r}0.162 \\
\end{array}$ \\
\hline 0.0679 & 0.406 & 6 & -0.011 & +0.103 \\
\hline 0.0769 & 0.353 & 3 & -0.006 & +0.072 \\
\hline 0.0882 & 0.289 & 7 & -0.004 & +0.036 \\
\hline 0.1016 & 0.234 & 5 & 0.000 & +0.013 \\
\hline 0.1179 & 0.182 & 11 & -0.001 & -0.001 \\
\hline 0.1382 & 0.131 & 11 & -0.007 & -0.007 \\
\hline 0.1642 & 0.086 & 11 & +0.001 & +0.001 \\
\hline 0.1886 & 0.044 & 11 & -0.001 & -0.001 \\
\hline 0.2225 & 0.007 & 12 & -0.001 & -0.001 \\
\hline 0.2514 & 0.000 & 12 & +0.002 & +0.002 \\
\hline 0.2757 & 0.004 & 12 & 0.000 & 0.000 \\
\hline 0.2965 & 0.023 & 12 & +0.002 & +0.002 \\
\hline 0.3152 & 0.040 & 12 & -0.003 & -0.003 \\
\hline 0.3374 & 0.080 & 12 & +0.001 & +0.001 \\
\hline 0.3699 & 0.145 & 12 & $\begin{array}{r}0.001 \\
\end{array}$ & +0.001 \\
\hline 0.4041 & 0.247 & 4 & +0.006 & +0.026 \\
\hline 0.4233 & 0.331 & 4 & +0.004 & +0.065 \\
\hline 0.4391 & 0.416 & 3 & +0.003 & +0.115 \\
\hline 0.4555 & 0.504 & 4 & -0.010 & +0.170 \\
\hline 0.4729 & 0.632 & 5 & +0.012 & +0.264 \\
\hline 0.4920 & 0.665 & 6 & +0.003 & +0.282 \\
\hline 0.5090 & 0.664 & 7 & +0.003 & +0.281 \\
\hline 0.5259 & 0.623 & 5 & -0.003 & +0.254 \\
\hline 0.5400 & 0.541 & 6 & -0.005 & +0.195 \\
\hline 0.5585 & 0.427 & 7 & -0.004 & +0.118 \\
\hline 0.5809 & 0.312 & 6 & +0.003 & +0.053 \\
\hline 0.5978 & 0.238 & 6 & +0.001 & +0.018 \\
\hline 0.6248 & 0.166 & 8 & +0.007 & +0.007 \\
\hline 0.6702 & 0.071 & 9 & +0.003 & +0.003 \\
\hline 0.7060 & 0.015 & 9 & -0.004 & -0.003 \\
\hline 0.7867 & 0.012 & 9 & 0.000 & -0.002 \\
\hline 0.8205 & 0.056 & 9 & +0.003 & +0.002 \\
\hline 0.8550 & 0.115 & 9 & -0.002 & -0.002 \\
\hline 0.8800 & 0.176 & 9 & +0.004 & +0.004 \\
\hline 0.8988 & 0.229 & 5 & -0.002 & +0.013 \\
\hline 0.9094 & 0.278 & 3 & +0.002 & +0.036 \\
\hline 0.9199 & 0.336 & 6 & +0.002 & +0.067 \\
\hline 0.9307 & 0.402 & 4 & 0.000 & +0.108 \\
\hline 0.9381 & 0.448 & 5 & -0.003 & +0.136 \\
\hline 0.9542 & 0.573 & 5 & +0.009 & +0.222 \\
\hline 0.9617 & 0.615 & 4 & -0.004 & +0.249 \\
\hline 0.9703 & 0.681 & 4 & +0.010 & $\begin{array}{r}+0.297 \\
\end{array}$ \\
\hline 0.9817 & 0.717 & 7 & $\begin{array}{r}0.007 \\
+0.007\end{array}$ & $\begin{array}{r}+0.318 \\
\end{array}$ \\
\hline 0.9984 & 0.718 & 8 & +0.001 & +0.313 \\
\hline
\end{tabular}
shown. In addition the normal points of the rectified light curve are given. The agreement is very satisfactory for stars of the W Ursae Majoris type.

TABLE II. Normal points of second light curve. 
TABLE III. Normal points of first light curve.

\begin{tabular}{|c|c|c|c|c|}
\hline Phase & $\begin{array}{c}\text { Obs. } \\
\Delta m\end{array}$ & $n$ & $O-C$ & $\begin{array}{l}\text { Rect. } \\
\Delta m\end{array}$ \\
\hline 0.0154 & 0.713 & 12 & +0.003 & +0.321 \\
\hline 0.0283 & 0.683 & 7 & +0.005 & $\begin{array}{l}+0.304 \\
\end{array}$ \\
\hline 0.0374 & 0.621 & 8 & -0.009 & +0.258 \\
\hline 0.0471 & 0.559 & 8 & -0.002 & +0.215 \\
\hline 0.0567 & 0.500 & 10 & +0.010 & +0.176 \\
\hline 0.0676 & 0.418 & 10 & +0.002 & +0.120 \\
\hline 0.0779 & 0.355 & 10 & +0.004 & +0.080 \\
\hline 0.0887 & 0.304 & 11 & +0.011 & +0.053 \\
\hline 0.1016 & 0.246 & 10 & +0.011 & +0.024 \\
\hline 0.1182 & 0.194 & 13 & +0.009 & +0.008 \\
\hline 0.1367 & 0.147 & 13 & 0.000 & 0.000 \\
\hline 0.1640 & 0.093 & 13 & -0.003 & -0.003 \\
\hline 0.1867 & 0.058 & 13 & -0.003 & -0.003 \\
\hline 0.2112 & 0.030 & 13 & -0.003 & -0.003 \\
\hline 0.2356 & 0.026 & 11 & +0.008 & +0.008 \\
\hline 0.2734 & 0.029 & 11 & +0.009 & +0.009 \\
\hline 0.3112 & 0.042 & 10 & -0.010 & -0.010 \\
\hline 0.3492 & 0.134 & 10 & +0.012 & +0.022 \\
\hline 0.4028 & 0.273 & 3 & +0.012 & +0.053 \\
\hline 0.4227 & 0.343 & 2 & -0.002 & +0.082 \\
\hline 0.4341 & 0.419 & 2 & +0.012 & +0.132 \\
\hline 0.4483 & 0.490 & 2 & 0.000 & +0.176 \\
\hline 0.4688 & 0.614 & 5 & -0.001 & +0.262 \\
\hline 0.4941 & 0.692 & 6 & +0.010 & +0.316 \\
\hline 0.5167 & 0.683 & 5 & +0.011 & +0.310 \\
\hline 0.5297 & 0.629 & 6 & +0.001 & +0.271 \\
\hline 0.5487 & 0.506 & 9 & -0.010 & +0.181 \\
\hline 0.5639 & 0.416 & 9 & -0.010 & +0.120 \\
\hline 0.5797 & 0.340 & 8 & -0.002 & +0.076 \\
\hline 0.5960 & 0.253 & 6 & -0.019 & +0.025 \\
\hline 0.6187 & 0.200 & 10 & -0.005 & +0.018 \\
\hline 0.6501 & 0.122 & 12 & -0.010 & +0.001 \\
\hline 0.6831 & 0.072 & 10 & +0.004 & +0.003 \\
\hline 0.7191 & 0.031 & 11 & -0.001 & -0.001 \\
\hline 0.7545 & 0.017 & 12 & -0.005 & -0.005 \\
\hline 0.7854 & 0.033 & 11 & -0.003 & -0.003 \\
\hline 0.8176 & 0.072 & 13 & +0.001 & +0.001 \\
\hline 0.8477 & 0.125 & 13 & +0.005 & +0.005 \\
\hline 0.8776 & 0.188 & 12 & +0.009 & +0.009 \\
\hline 0.8976 & 0.244 & 4 & +0.015 & +0.025 \\
\hline 0.9107 & 0.284 & 4 & +0.003 & +0.037 \\
\hline 0.9225 & 0.355 & 5 & -0.001 & +0.080 \\
\hline 0.9347 & 0.425 & 5 & -0.005 & +0.123 \\
\hline 0.9436 & 0.485 & 5 & -0.008 & +0.163 \\
\hline 0.9528 & 0.561 & 7 & -0.007 & +0.217 \\
\hline 0.9621 & 0.613 & 7 & -0.016 & +0.252 \\
\hline 0.9717 & 0.680 & 9 & +0.001 & +0.301 \\
\hline 0.9837 & 0.710 & 12 & +0.004 & +0.319 \\
\hline 0.9982 & 0.710 & 12 & -0.009 & +0.315 \\
\hline
\end{tabular}

Radial velocity curves for RZ Comae were published by Struve and Gratton (1948) from 24 spectra. A spectral type of $\mathrm{K} 0$ was determined. The spectroscopic and photometric elements were combined to give the following results:

$$
\begin{array}{rlrl}
a_{1} & =620000 \mathrm{~km} & a_{2} & =1270000 \mathrm{~km} \\
a_{g} & =1.31 \odot & a_{s} & =0.70 \odot \\
b_{g} & =1.11 \odot & b_{s} & =0.60 \odot \\
\mathfrak{M}_{g} & =1.59 \odot & \mathfrak{M}_{s} & =0.77 \odot \\
\rho_{g} & =0.98 \odot & \rho_{s} & =3.06 \odot \\
P \sqrt{\rho_{g}} & =0.33 & P \sqrt{\rho_{s}} & =0.59
\end{array}
$$

It is very probable that the geometrical elements of the system did not change in the short time interval elapsing between the two curves. The surface brightnesses may have varied, however. The elements obtained from the second light curve can now be used to explain the first light curve (JD 2436220-285). The rectified normal points of primary minimum agree very well with the theoretical curve. This was expected since the two light curves are almost identical during primary eclipse. However, the rectified normals of secondary minimum are systematically fainter by $0 \mathrm{~m} .02$ than the predicted theoretical light curve.

In Table III the observed normals of the first light curve are compared with the theoretical values. The latter were corrected for the systematic deviation occurring during secondary minimum. The $O-C$ 's are shown. In addition the normal points of the rectified light curve are given.

In Fig. 1 the theoretical light curves have been drawn. The orientation of the system at different phases is also shown. During primary eclipse that hemisphere of the larger star which is not facing the smaller star is visible. No noticeable change occurred in the surface brightness of this hemisphere since the light curves are practically identical during this eclipse. Outside eclipses and during secondary eclipse the first light curve is fainter. A subluminous region which is partially visible can account for this deviation.

Broglia assumed that there had been a change in the surface brightness of the external hemisphere of the smaller component visible at secondary eclipse. However, a subluminous region on the facing side of the larger star can also explain the deviation. This last identification of the subluminous region explains two more details. At phase 0.55 the two light curves are almost identical. Consequently the smaller star must then eclipse most of the subluminous area on the larger star. This region must therefore be somewhat unsymmetrically placed with respect to the endpoint of the larger star. Moreover, outside eclipse the smaller and hotter star receives less light from the larger star to reflect to the earth. Thus the reflection coefficient of the hotter star will decrease while the reflection coeffcient of the cooler star will remain the same. This means that one can expect a larger negative value for $A_{1}$. This was indeed found for the first light curve.

\section{ACKNOWLEDGMENTS}

Part of this work was supported by a grant from the National Science Foundation.

\section{REFERENCES}

Broglia, P. 1960, Contributi Milano-Merate, No. 165. Struve, O., and Gratton, L. 1948, Astrophys. J. 108, 497. 